\title{
Optimal Location and Minimum Number of Superconducting Fault Current Limiters for the Protection of Power Grids
}

\author{
Xiuchang Zhang ${ }^{\mathrm{a}}$, Jianzhao Geng ${ }^{\mathrm{a}}$, T. A. Coombs ${ }^{\mathrm{a}}$, H. S. Ruiz ${ }^{\mathrm{b}}$ \\ ${ }^{a}$ Department of Engineering, University of Cambridge, 9 JJ Thomson Avenue, Cambridge, CB3 OFA, U.K. \\ ${ }^{b}$ Department of Engineering, University of Leicester, University Road, Leicester, LE1 7RH, U.K.
}

\begin{abstract}
This paper presents a novel method to determine the optimal strategy for the allocation of multiple resistive superconducting fault current limiters (SFCLs) aiming to improve the overall protection of standard power grids. The presented approach allows for the straightforward determination of the optimal resistance of the SFCL, accounting for short circuit events occurring at different locations, by modelling the electro-thermal properties of the SFCL via a temperature dependent E-J power law. This material law, based on previous experimental evidence, allows for the introduction of flux pinning, flux creep, and flux flow properties of the superconducting material within a minimum level of complexity. Thereby, we have observed a distinctive kink pattern in the current limiting profiles of the SFCLs, from which no further reduction of the first peak of the fault current is achieved when a greater resistance is considered, allowing a univocal determination of the optimum SFCL resistance. This peculiarity is not observed when the model for the quench properties of the SFCL is simplified towards an exponential resistance, although the last can be used as an auxiliary process for addressing the first guess on the resistance value required for a specific strategy, as it demands less computing time. We have also determined that for many of the cases studied, i.e, for the combinations between one or more SFCLs installed at different locations, and those subjected to fault events located at different points in the network, the recovery time of the superconducting properties of at least one of the SFCLs can last for more than five minutes, constraining the feasibility of a large-scale deployment of this technology. However, by assuming that the practical operation of the SFCL is assisted by the automatic operation of a bypass switch when the SC material is fully quenched, we have determined that the optimal strategy for the overall protection of power grids of standard topology requires a maximum of three SFCLs, with recovery times of less than a few seconds. This information is of remarkable value for power system operators, as it can establish a maximum investment threshold which ultimately can facilitate making decisions regarding the deployment of SFCL technologies.
\end{abstract}

Keywords:

Superconducting fault current limiter, Fault protection, Optimal location, Electrical power grid, Optimal resistance. PACS: 84.71.-b, 85.25.Am, 88.05.Bc, 88.05.Ec, 88.05.Lg, 88.80.Cd, 88.80.H, 88.50.Mp

\section{Introduction}

The expansion of distributed generation, grid interconnection, and the continuously growing demand for electric power are just some of the many factors that have led power network operators to develop critical reports, addressing the actual need for large scale upgrading of conventional schemes of fault protection. [1]. Various strategies for mitigating fault current levels are commonly implemented in the power industry, where

Email addresses: xz326@cam.ac.uk (Xiuchang Zhang), dr.harold.ruiz@leicester.ac.uk (H. S. Ruiz) the most conventional ones include the construction of new substations, splitting existing substation busses, the upgrading of circuit breakers, and/or the installation of three-winding transformers. Nevertheless, all these operational practices imply a non negligible degradation of the reliability figures of the power system under actual operating conditions, which ultimately may involve significant economic losses and hence the need for further investment [2]. Series reactors and solid state fault current limiters are also common strategies for reducing the fault levels in existing power grids, although these devices cause a noticeable voltage drop and therefore considerable power losses during the normal operation of 


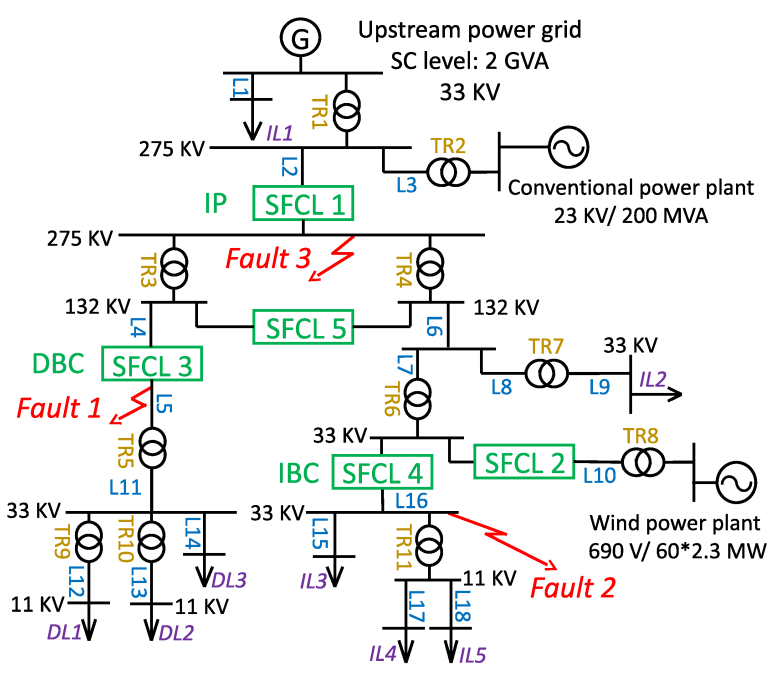

Figure 1: Single-line diagram of the power system layout considered as the case study.

the network [4, 5]. However, under normal operational conditions of the power network, novel technologies such as superconducting fault current limiters (SFCLs), with their unique property of nearly zero electrical resistance, offer the possibility to improve voltage stability, power supply, quality, and overall efficiency of the electric grid without the need for constructing additional substations or adding new infrastructure [6, 7, 8, 9].

When a short circuit event or electric fault occurs, the SFCL is custom designed in order to trigger the fast transition from its superconducting state to the so-called quenched state. The quench state is characterised by a high electrical resistivity, aiming to limit the first peak of the fault current to acceptable operational levels, either by ensuring the automatic recovery of the protection scheme with no disruptions on the supply, or by allowing enough time for the prompt activation of conventional protection systems such as circuit breakers [6]. Commonly, fault events generate voltage sags lasting between 0.5 and 60 cycles, affecting consumers differently according to their location in the electric network. For instance, even though the load current at certain locations is small compared to the fault current, changes in the load current during and after the fault strongly influence the voltage at the power equipment terminals, where about $85 \%$ of power supply malfunctions are caused by voltage sags or interruptions of fewer than $1 \mathrm{~s}$ duration, led by the occurrence of a fault event at some branch of the power network [3].

Thus, in order to determine the optimum performance of power systems from the protection scheme view-
Table 1: Parameters of loads, transformers and transmission lines

\begin{tabular}{lccccccc}
\hline \multicolumn{2}{l}{ Loads (MW) } & \multicolumn{2}{c}{ Transformers (MVA) } & \multicolumn{4}{c}{ Transmission Lines $(\mathrm{km})$} \\
\hline DL1 & 20 & TR1\&2 & 250 & L1\&8 & 4 & L11 & 12 \\
DL2 & 30 & TR3\&5 & 220 & L2 & 70 & L12 & 2 \\
DL3 & 50 & TR4\&6 & 200 & L3\&4 & 20 & L13 & 1 \\
IL1 & 80 & TR7 & 120 & L5 & 30 & L14 & 6 \\
IL2 & 70 & TR8 & 180 & L6 & 80 & L15 & 5 \\
IL3 & 55 & TR9 & 40 & L7 & 10 & L16 & 15 \\
IL4 & 30 & TR10 & 60 & L9 & 1 & L17 & 4 \\
IL5 & 30 & TR11 & 90 & L10 & 10 & L18 & 2 \\
\hline \hline
\end{tabular}

point, there is no $100 \%$ reliable solution for determining the optimal location for the installation of a single SFCL, even though it may be based upon topographical studies of fault detection. However, solutions can be provided for isolated scenarios [10, 11, 12], although a detailed analysis is necessary to select the correct and most economical solution for a particular situation (fault location, particular network, and SFCL location). Nevertheless, for each scenario and with a predefined location for installing the SFCL, it is possible to study the reliability figures of the protection scheme, by analysing the multiple locations where a fault event may occur, i.e, the first peak limiting performance is assessed at the different busbars of a power network under different fault events. Then, once the most severe cases for fault protection have been identified, multiple SFCLs can be installed at the different branches of the power network aiming to protect, simultaneously, all the distribution branches. Thus, in this paper we demonstrate how the optimal strategy for the overall protection of standard power networks, under the protection scheme implemented by the integration of SFCLs, implies a maximum investment of three SFCLs installed at specific locations regardless of the ubiety of the fault event.

This paper is organised as follows. Section 2 presents the main features of the simulated power grid, the minimum physical characteristics that need to be considered for modelling the quench properties of the superconducting material, and the fault current limiting dynamics under multiple protection schemes and load flow analysis, i.e., scrutinising different locations for the optimal installation of an SFCL in conjunction with the occurrence of fault events at diverse locations. Then, in Section 3 we demonstrate the existence of a threshold value on the number of SFCLs needed for the overall protection of the power network, based upon a comprehensive study of the number of scenarios where the allocation of multiple SFCLs and the concurrent occurrence of fault events can be considered. Finally, the main conclusions 


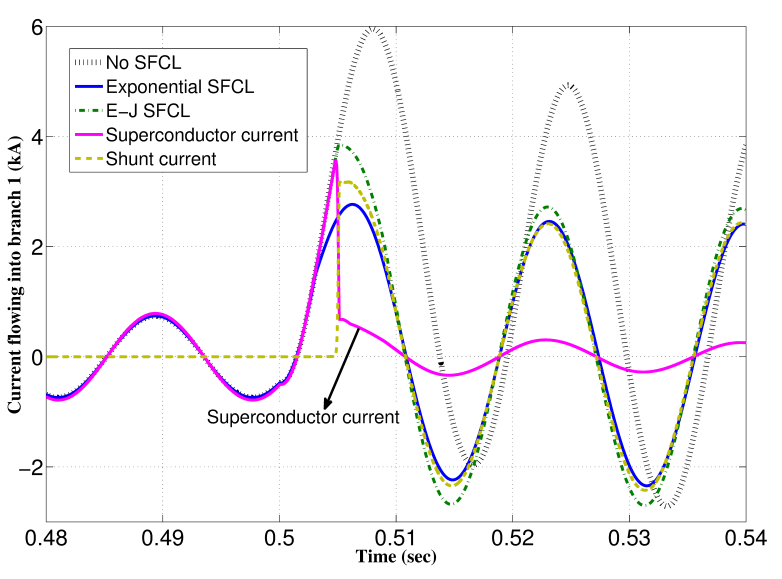

Figure 2: (Colour Online) Fault-current limiting dynamics of SFCL-3 responding to Fault-1 (see Fig. 1 under $e$ law and $E-J$ power law models. The current sharing profile between the SC and the shunt resistance of the $E-J$ power law SFCL model is also displayed.

of this paper are presented in Section 4

\section{Power System Configuration and SFCL Scheme}

For the purposes of this study the simulated power system is designed within UK network standards [13], and is represented by the single-line diagram shown in Fig. 1. The power system includes a 200 MVA conventional power plant connected to a 2 GVA short circuit rated upstream power grid which supplies an interconnected $80 \mathrm{MW}$ industrial load (IL1). Then, the power flow continues downwards to feed domestic and industrial networks, characterised by three domestic loads (DL1-DL3) and four industrial loads (IL2-IL5) (see also Table 11). Distributed renewable energy systems such as onshore wind power plants have also been considered under the scope of the simulated power system. For the case study presented in this manuscript, the wind power plant has been simulated in accordance with the power specifications provided by the Phase II Crystal Rig wind farm in East Lothian, Scotland, giving a nameplate capacity of $138 \mathrm{MW}$ (60 Siemens $2.3 \mathrm{MW}$ turbines) [14].

Concerning fault analysis, it is well known that the maximum reliability of a protection scheme needs to be assessed in terms of the prospective occurrence of symmetric three-phase short circuit faults, these causing a severe decrease on the power system impedance. Thus, by considering the most hazardous scenarios for the operation of the system, three prospective locations were simulated for three-phase to ground short circuit events representing contingencies at the domestic network (Fault 1), the industrial network (Fault 2), and the high voltage transmission lines (Fault 3). The study takes into account the fault current dynamics for overall protection schemes, assuming an investment of up to five SFCLs to be installed at different locations, and tested to stand the occurrence of severe fault events. Thus, each combination between the occurrence and location of a fault event together with the allocation of a sole or multiple SFCLs, may define a singular protection scheme, which does not necessarily protect the stability of the overall power grid. Therefore, by analysing the topology of the power system shown in Fig. 1 the following five locations have been assumed to be the most suitable integration points for the installation of an SFCL, namely: the outgoing feeder of the conventional power plant (SFCL 1), the outgoing feeder of the wind farm (SFCL 2), the ports of the domestic (SFCL 3) and industrial (SFCL 4) branches, and the bus-tie coupling the domestic and industrial networks (SFCL 5). The locations SFCL1 and SFCL 2 have been chosen because when the SFCL is installed at a feeder location, the need for upgrading interconnected substations due to the added generation capacity and the increase of the short-circuit current levels can be postponed, resulting in high economical savings [15]. On the other hand, it is a common practice to assume that most of the fault events occur at the domestic branch connection (DBC) and/or at the industrial branch connection (IBC) [8, 10]. The main advantage of installing SFCLs at these ports, SFCL3 and SFCL4 respectively, is the possibility to increase the short-circuit capacity of the subgrids during normal operation without increasing the fault currents. Finally, the installation of a SFCL in the busbar coupling at transmission voltage levels (SFCL5), aims to provide a flexible operation of the transformers TR3 and TR4 while reducing fault currents to acceptable levels, complying with the common practice of redundancy for feeding a distribution grid.

Concerning to the electrical properties that define the practical operation of the SFCL under the scope of load flow analysis, the quench transition of the SFCL is equivalent to considering the sudden insertion of a high impedance during, and after the occurrence of a fault event $\left(t=t_{f}\right)$. In fact, during the normal operation of the power system, i.e., before the occurrence of a fault $\left(t<t_{f}\right)$, and after its full clearance and later recovery of superconducting properties $\left(t \geq t_{f c}+t_{r}\right)$, the resistive impedance of the SFCL is nearly invisible to the load flow. Therefore, it is reasonable to assume that under these conditions the SFCL acts as a small resistance defined by $R_{n}=10^{-6} \Omega[10,16]$. Then, the fault pro- 
tection capabilities of the SFCL are captured by modelling the quenching properties of the superconducting material beyond the steady-state conditions [12], where the time-dependance comes into play either via a simplified exponential function for the resistance dynamics of the SFCL under fault conditions [9, 17, 18], or a temperature dependent $E-J$ power law describing the flux-creep and flux-flow properties of the superconducting material [19, 20]. In the first model, once the SFCL detects the occurrence of a fault event, its nominal resistance swiftly increases towards the maximum resistance of the quenched material $\left(R_{m}\right)$ until the clearance of the fault at $t=t_{f c}$ is achieved, i.e.,

$$
R(t)=R_{m}\left[1-\exp \left(-\frac{t-t_{f}}{t_{s c}}\right)\right], \in \text { Fault time }
$$

where the upper limit $t=t_{f c}$ stands for the recovery period of the superconducting material $\left(t_{f c} \leq t<t_{f c}+t_{r}\right)$, and $t_{s c}$ defines the minimum quench time allowing for the propagation of the normal zone over the entire superconductor $(\sim 1 \mathrm{~ms})$. However, in order to attain a more precise validation of the SFCL protection scheme regarding safety operation and power stability conditions, the recovery time of the SFCL cannot be assumed as an ad hoc parameter. Therefore, besides the exponential model, we have also studied the impact of considering the temperature dependence of the flux-creep and flux-flow regimes describing the time-dependent quench of the superconducting material, such that the averaged instantaneous temperature of the SFCL, $T(t)$, can be calculated by solving the classical heat transfer equation [21]. Theis thermal subsystem is then coupled to the electrical module of the power system, as a temperature dependent $E-J$ power law of similar structure to the one proposed by Lacroix and Sirois in Ref. [20]. In our case, a cylindrical bulk of Bi2212 immersed in a liquid nitrogen bath at $1 \mathrm{~atm}$ pressure $\left(T_{0}=77 \mathrm{~K}\right)$ has been assumed, leading to:

$$
E(T, t)=E_{0} \cdot\left[\frac{J(t)}{J_{c}\left(T_{0}\right)} \frac{\left(T_{c}-T_{0}\right)}{\left(T_{c}-T(t)\right)}\right]^{n(T, t)} \forall T(t)<T_{c},
$$

with

$$
n(T, t)=\left(n_{0}-1\right)\left(\frac{T_{c}-T(t)}{T_{c}-T_{0}}\right)^{1 / 4}+1,
$$

for $T_{c}=85 \mathrm{~K}, E_{0}=1 \mu \mathrm{V} / \mathrm{cm}, J_{c}\left(T_{0}\right)=12 \mathrm{MA} / \mathrm{m}^{2}$, and $n_{0}=9$, in good agreement with the experimental results reported in Refs. [22, 23, 24, 25, 26]. Then, the normal properties of the superconducting material for $T>T_{c}$ are simulated as $E(T, t)=\rho\left(T_{c}\right) J(t)\left(T(t) / T_{c}\right)$, with $\rho\left(T_{c}\right)=7 \times 10^{-6} \Omega$.

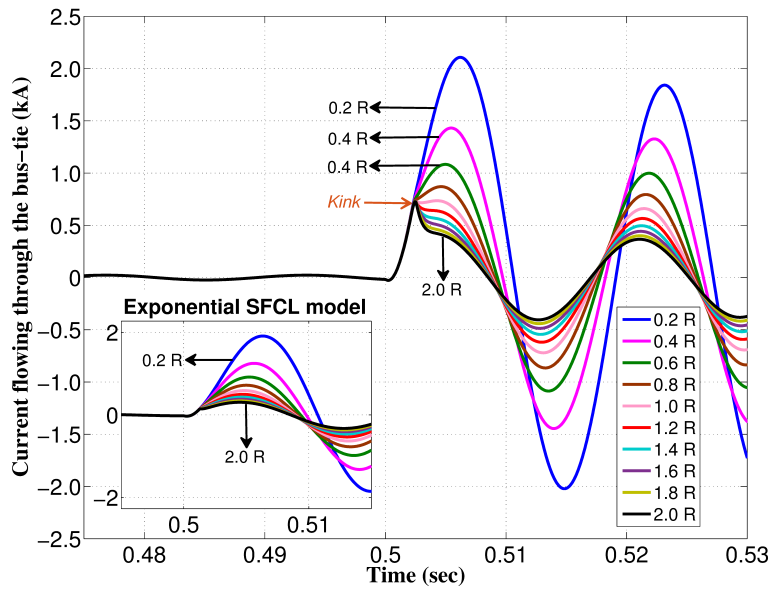

Figure 3: (Colour Online) Limited current of SFCL-5 responding to Fault-2 (see Fig. 1) as a function of its maximum rated resistance $R_{m}=$ $0.2 R, 0.4 R, \ldots, 2 R$, with $R=R_{m}^{\dagger}=25 \Omega$, under the $e$-law (inset) and the $E-J$ power law models.

Finally, in order to protect the superconducting components from the uncontrolled occurrence and propagation of hot spots during fault events, the recovery characteristics of the SFCL are assisted by the parallel connection of a shunt resistance, $R_{s}$, acting as an electrical bypass during the quench regardless of the material law governing the transition of the superconducting state into the normal state of the superconductor. Thus, based upon the technical characteristics of the power system described above, and the physical properties emulating the behaviour of the SFCL, the main results derived from our study are presented below.

\section{Performance analysis of the SFCL scheme for the overall protection of the power grid}

In order to illustrate the reliability figures that may support investment in SFCL technologies, in this section we present a comprehensive study of the current limiting performance for the action of one or multiple SFCLs subjected to the occurrence of different fault events, and the identification of their optimal location.

In Fig. 2, the results obtained for a three-phase-toground fault at the domestic branch (Fig. 1, Fault 1), with an SFCL either modelled as a time-dependent exponential resistor or by means of the temperaturedependent $E-J$ power law describing the quench properties of the superconducting material, are shown when the SFCL is installed in between transmission lines L3 and L4, and the fault lasts for $200 \mathrm{~ms}$. After following the steady state behaviour of the power system, the 
Table 2: Calculated recovery time, $t_{r}$, of the SFCLs depicted in Fig. 1 with $\left({ }^{*}\right)$ and without $\left(^{\ddagger}\right)$ the assistance of the BS strategy.

\begin{tabular}{lcccccc}
\hline \hline & Fault 1 $^{(*)}$ & Fault $1^{(\ddagger)}$ & ${\text { Fault } 2^{(*)}}$ & Fault 2 & Fault $3^{(*)}$ & Fault $3^{(\ddagger)}$ \\
\hline SFCL 1 & $0.71 \mathrm{~s}$ & $329 \mathrm{~s}$ & N/A $^{\S}$ & N/A & $0.73 \mathrm{~s}$ & $370 \mathrm{~s}$ \\
SFCL 2 & $1.34 \mathrm{~s}$ & $482 \mathrm{~s}$ & $1.55 \mathrm{~s}$ & $529 \mathrm{~s}$ & $1.63 \mathrm{~s}$ & $559 \mathrm{~s}$ \\
SFCL 3 & $0.87 \mathrm{~s}$ & $320 \mathrm{~s}$ & N/A $^{\S}$ & N/A & N/A & N/A \\
SFCL 4 & N/A $^{\S}$ & N/A & $2.11 \mathrm{~s}$ & $729 \mathrm{~s}$ & N/A & N/A \\
SFCL 5 & $0.87 \mathrm{~s}$ & $1.01 \mathrm{~s}$ & $0.80 \mathrm{~s}$ & $0.82 \mathrm{~s}$ & $0.77 \mathrm{~s}$ & $0.79 \mathrm{~s}$ \\
\hline \hline
\end{tabular}

$\$$ N/A stands for those occasions where the fault event does not represent a hazard to the network where the SFCL is installed, and consequently the quenching of the SFCL is not triggered under these conditions.

fault is initialised at $t_{f}=0.5 \mathrm{~s}$, where the first peak of the prospective fault current measured without the insertion of the SFCL (6 kA) is effectively limited to $2.7 \mathrm{kA}$ when the exponential model is considered, i.e., attaining a $55 \%$ reduction on the first peak of the fault current, whilst only about a $35 \%$ current reduction is achieved with the temperature-dependent $E-J$ power law. This result indicates that although the simplified exponential resistance model can overestimate the rate of change for the resistance curve of the SFCL, the minimum rated resistance for fault protection, $R_{m}$, can be estimated from the exponential model if the minimum percentage of current reduction is defined. This value can therefore be used as a control asset for defining the actual impact of the SFCL protection scheme when considering more sophisticated models like the temperature dependent $E-J$ power law. In fact, after a thorough comparison of the fault current limiting performance with the exponential resistance model and the temperature dependent $E-J$ power law model, we have observed that a univocal $R_{m}^{\dagger}$ value can be identified via the striking occurrence of a "kink" pattern on the SFCL's current that only appears when the $E-J$ power law is considered. The generic relationship between $R_{m}^{\dagger}$ and the reduction on the first peak of the fault current can be observed in Fig. 3 .

Fig. 3 displays the profile of current flowing through an SFCL installed at the bus-tie coupling the domestic and industrial networks (SFCL 5), when a $200 \mathrm{~ms}$ threephase to ground fault in the domestic network (Fault 2) is initialised at $t_{f}=0.5 \mathrm{~s}$. Under the normal operation of the power grid, the overall system has been regulated such that only a current of $20 \mathrm{~A}$ is observed at the bustie. However, when a fault event occurs at the industrial network (Fault 2), the first peak of the short circuit current can reach up to $4.1 \mathrm{kA}$. Thus, when the SFCL exponential model has been considered, a minimum $R_{m}$ value of $5 \Omega$ is assumed as it shows a current reduction of $\sim 55 \%$ over the first peak of the fault current. Then, $R_{m}$ is gradually increased up to $50 \Omega$, attaining a reduction of about $93 \%$ on the first peak of the fault current
Table 3: Table of protection strategies, $-s-$, accounting for the deployment of up to five SFCLs.

\begin{tabular}{cccccc}
\hline \hline$-s-$ & SFCLs & $-s-$ & SFCLs & $-s-$ & SFCLs \\
\hline $\mathbf{1}$ & 1 & $\mathbf{1 1}$ & 2,4 & $\mathbf{2 1}$ & $1,4,5$ \\
$\mathbf{2}$ & 2 & $\mathbf{1 2}$ & 2,5 & $\mathbf{2 2}$ & $2,3,4$ \\
$\mathbf{3}$ & 3 & $\mathbf{1 3}$ & 3,4 & $\mathbf{2 3}$ & $2,3,5$ \\
$\mathbf{4}$ & 4 & $\mathbf{1 4}$ & 3,5 & $\mathbf{2 4}$ & $2,4,5$ \\
$\mathbf{5}$ & 5 & $\mathbf{1 5}$ & 4,5 & $\mathbf{2 5}$ & $3,4,5$ \\
$\mathbf{6}$ & 1,2 & $\mathbf{1 6}$ & $1,2,3$ & $\mathbf{2 6}$ & $1,2,3,4$ \\
$\mathbf{7}$ & 1,3 & $\mathbf{1 7}$ & $1,2,4$ & $\mathbf{2 7}$ & $1,2,3,5$ \\
$\mathbf{8}$ & 1,4 & $\mathbf{1 8}$ & $1,2,5$ & $\mathbf{2 8}$ & $1,2,4,5$ \\
$\mathbf{9}$ & 1,5 & $\mathbf{1 9}$ & $1,3,4$ & $\mathbf{2 9}$ & $1,3,4,5$ \\
$\mathbf{1 0}$ & 2,3 & $\mathbf{2 0}$ & $1,3,5$ & $\mathbf{3 0}$ & $2,3,4,5$ \\
& & & & $\mathbf{3 1}$ & 12345 \\
\hline \hline
\end{tabular}

with a backwards displacement of the peak values. On the other hand, when the same resistance values are considered for the definition of the normal properties of the superconducting material (after a full quench), but with the flux creep and flux flow phenomena taken into consideration by the temperature dependent E-J power law, a quite distinctive feature at $0.7 \mathrm{kA}$, here called "kink", has been observed for SFCLs with $R_{m}>25 \Omega$.

The appearance of this striking feature on the current limiting profiles of the SFCL scheme implies the existence of an optimal resistance value, $R_{m}^{\dagger}$, equal to $25 \Omega$ for the case displayed in Fig. 3. from which no further reduction on the first peak of the fault current can be achieved. It is noteworthy that the identification of $R_{m}^{\dagger}$ via power flow analysis provides a simplified but straightforward way of determining the minimum amount of superconducting material that is needed for the fabrication and scalability of a resistive type SFCL tailored to different grids. However, although it is true that the installation of an SFCL can be considered as a reliable protection scheme in terms of fault current reduction, and the manufacturing costs can be reduced once $R_{m}^{\dagger}$ has been identified, the actual costs of operation of the SFCL cannot be estimated until the recovery time of the SFCL is adequately assessed.

It is possible to demonstrate that, after the full clearance of a fault event lasting a few tens of milliseconds, the SFCL may require several minutes to stabilise its temperature and hence recover its SC properties. This ultimately compromises the deployment and reliability of the SFCL protection scheme. However, in order to decrease the recovery time of the SFCL to less than $3 \mathrm{~s}$ regardless of the SFCL location and where the fault current event occurs, it is possible to improve the SFCL protection scheme by connecting a bypass switch paral- 

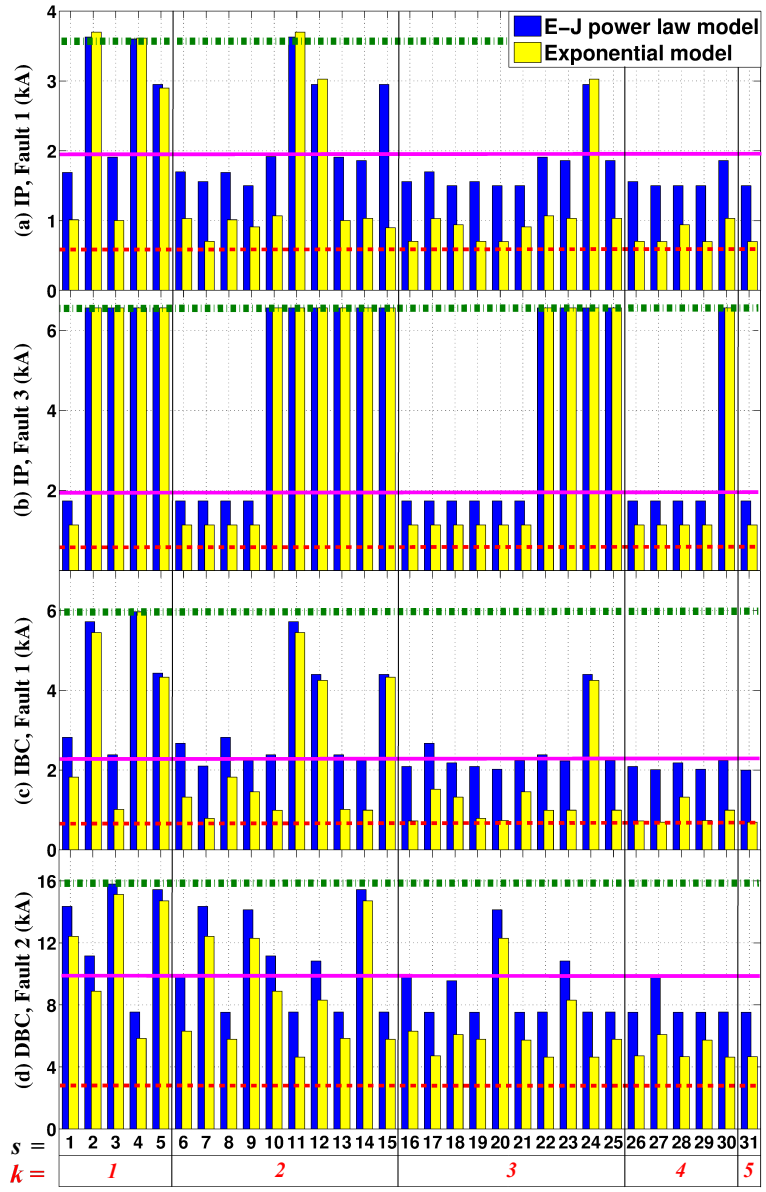

Figure 4: (Colour Online) First peak limiting performance of the 31 installation strategies. Results are shown only for the most hazardous measurement conditions identified during the analysis. The green dash-dotted lines show the prospective fault current levels without the SFCLs. The red dashed lines represent the current levels for the normal operation of the power grid $\left(I_{n}\right)$, and the threshold value for safety regulation $\left(I_{s r}\right)$ is defined as three times this value (purple solid lines) as common practice [19].

lel to the terminals of the SFCL. The bypass switch (BS) allows for the electrical isolation of the SFCL from the power grid after the fault event has been cleared, hence reducing the Joule heating on the SC material and therefore its recovery time $t_{r}$. Another advantage of the BS scheme is that it allows the automatic reconnection of the SFCL to the live power grid once the SC state is recovered [27]. Without the external assistance of the BS, and for fault events triggering the protection characteristics of the SFCL, we have determined that the recovery time of the SC properties can take over five minutes in most cases (see Table 2).

Thus, once the optimal resistance $R_{m}^{\dagger}$ has been identi-
Table 4: Operational safety margin (OSM) for the $-s-$ protection strategies, which consider the installation of up to 3 SFCLs.

\begin{tabular}{ccccc}
\hline \hline$s$ & IP-Fault 1 & IP-Fault 3 & IBC-Fault 1 & DBC-Fault 2 \\
\hline $\mathbf{1 6}$ & $16 \%$ & $10 \%$ & $9 \%$ & $-1 \%$ \\
$\mathbf{1 7}$ & $8 \%$ & $10 \%$ & $-15 \%$ & $24 \%$ \\
$\mathbf{1 8}$ & $18 \%$ & $10 \%$ & $4 \%$ & $3 \%$ \\
$\mathbf{1 9}$ & $16 \%$ & $10 \%$ & $9 \%$ & $24 \%$ \\
$\mathbf{2 0}$ & $18 \%$ & $10 \%$ & $13 \%$ & $-41 \%$ \\
$\mathbf{2 1}$ & $18 \%$ & $10 \%$ & $-2 \%$ & $24 \%$ \\
$\mathbf{2 2}$ & $3 \%$ & $-233 \%$ & $-4 \%$ & $24 \%$ \\
$\mathbf{2 3}$ & $5 \%$ & $-233 \%$ & $2 \%$ & $5 \%$ \\
$\mathbf{2 4}$ & $-53 \%$ & $-233 \%$ & $-48 \%$ & $24 \%$ \\
$\mathbf{2 5}$ & $5 \%$ & $-233 \%$ & $-2 \%$ & $24 \%$ \\
\hline \hline
\end{tabular}

fied, and the BS strategy has been incorporated, the reliability analysis for the overall protection of the power grid lies in the accurate assessment of the optimal location for the installation of an SFCL, and presumably the need for considering adding more than one SFCL. In fact, as the location where a fault event is likely to occur is unpredictable, and the current manufacturing costs of an SFCL are not currently competitive against traditional protection schemes [28], the distribution network operators are reluctant to invest in large scale deployment of SFCL technologies, if there is not a clear threshold for the actual number of SFCLs required for the overall protection of the grid, and if the optimal locations where these SFCLs should be installed are unknown. Therefore, in order to determine the optimal strategy for the installation of one or multiple SFCLs, and thereby ensure the overall protection of the power grid under diverse fault conditions, a set of five installation points were chosen after analysing the capability of each individual SFCL by limiting the fault current to within the rating of existing switchgear. The optimal SFCL protection scheme is determined from the power flow analysis of the 31 possible strategies, $s$, that combine the five SFCL locations (see Table 3). Each strategy was assessed under all three fault conditions illustrated in Fig. 1, resulting in the study of 93 different cases.

By scrutiny of the most hazardous fault events in each of the aforementioned 93 cases, Fig. 4 shows the obtained results for the reduction of the first peak of the fault current from the analysis of the 31 protection strategies. In particular, we refer to the measuring points located at (see Fig 1): (a) the integration point (IP) with a fault event at the domestic network (Fault 1), (b) the IP with a fault event at the high voltage transmission line (Fault 3), (c) the domestic branch connection (DBC) 
with a fault event in the domestic network (Fault 1), and (d) the industrial branch connection (IBC) with a fault event in the industrial network (Fault 2).

The extensive set of results derived from this study can be simplified by calculating the operational safety margin (OSM) for each one of the 31 protection strategies, i.e., OSM $=\left(I_{s r}-I_{l}\right) /\left(I_{s r}\right) \times 100 \%$, with $I_{l}$ being the magnitude of the first peak of the limited current, and the safety threshold $I_{s r}=3 I_{n}$ (see Fig. 4). Thus, when the protection strategy produces a positive impact on all the measuring points, the strategy is considered to be highly suitable, and eventually it can be considered optimal depending on the specific demands of the power industry. In the presented case, if the effectiveness of a protection strategy that assumes a $k$-number of SFCLs is within $10 \%$ of the effectiveness of any of the other protection schemes with $k-1$ SFCLs, the last is considered to be the optimal protection scheme. The overall algorithm for identification of the optimal protection scheme is illustrated in Fig. 5 .

In all cases, the fault has been initiated after 25 cycles of normal operation $\left(t_{f}=0.5^{\prime \prime}\right)$, with a duration of 10 cycles $\left(t_{f c}=0.7^{\prime \prime}\right)$ and, the computation has been carried up to $t=4^{\prime \prime}$ which is generally greater than $t_{f c}+t_{r}$ when the BS has been included. For the installation of only one SFCL $k=1$, the mean computing time for the subset of strategies $s \in[1: 5]$ is $\sim 58 \pm 5^{\prime \prime}$ regardless of the location of the fault, with an average increment of $\sim 18^{\prime \prime}$ for each strategy $(s)$ with an added SFCL $(k+1)$. The actual total computing time for the 93 studied cases is of $8073.51^{\prime \prime}$, i.e., less than 2 hours and 15 minutes.

We have therefore determined that in order to protect the overall grid from any of the fault events hereby considered, the installation of three SFCLs is enough within the established safety thresholds (see Fig. 4). In particular, only two different arrangements made of up to three SFCLs have accomplished the desired conditions (see Table 4, i.e., the strategies $s=18$ and $s=19$, with the SFCLs installed at locations $[1,2,5]$, and $[1,3,4]$, respectively. It is to be noted that although strategy 19 , which involves installing the SFCLs at the outgoing feeder of the power plant and the feeding ports of the domestic and industrial networks, offers a significant improvement when the fault occurs at the domestic branches, it is necessary to consider the needs of expansion of the power grid when making decisions. Thus, we conclude that strategy 18 which rather of consider installing SFCLs at locations 3 and 4, it allows for direct protection of the wind power plant with an SFCL installed at location 2, therefore preventing potential islanding problems [29], and the other SFCL installed at the bustie coupling the domestic and industrial networks (Lo-

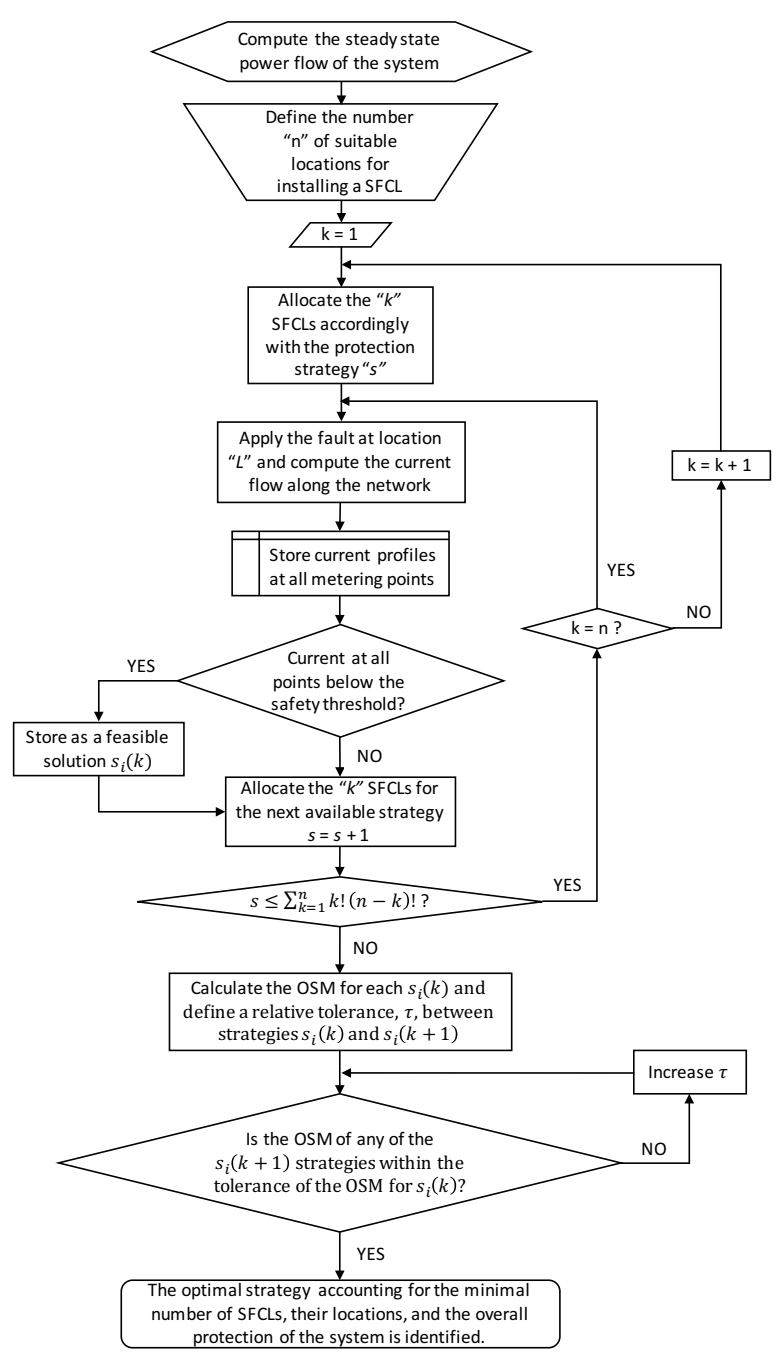

Figure 5: Flowchart of the algorithm for the identification of the optimal SFCL strategy.

cation 5), is the optimal protection scheme for the deployment of the SFCLs. In fact, introducing a SFCL at the bus-tie (SFCL 5) would enable one to operate the transformers TR3 and TR4 in parallel, resulting in a doubled short circuit capacity and lower transformer losses [30], which in turn results in lower voltage drops, improved stability, and savings in load connection expenses [15].

\section{Conclusion}

A comprehensive method for determining the optimal strategy for the integration of SFCLs in standard power networks has been presented. The method allows for calculating the minimum number of SFCLs which 
need to be deployed for the overall protection of the network. In order to determine the optimal resistance of the SFCL, two different material laws for defining the electrical behaviour of the SC material have been considered. Firstly, an exponential resistance model for modelling the quench properties of the superconducting material without expressly accounting for temperature gradients has been invoked, simultaneously defining the reference point for determining a minimum threshold for the SFCL resistance, $R_{m}$, which may lead to the desired limitation of the fault current. Secondly, supported on the experimental observations reported in the literature, a more realistic temperature-dependent $E-J$ power law model was implemented. From this, a distinctive kink pattern on the profiles of limiting current allowed us to establish a univocal method for the determination of the optimal resistance, $R_{m}^{\dagger}$, of each one of the SFCLs installed.

Thus, once $R_{m}^{\dagger}$ was determined for the different locations (L) where an SFCL might be placed, accounting for fault events occurring at different locations, the complete number of protection strategies were identified, being the possible combinations between a sole SFCL, or multiple SFCLs each installed at one of the $L$ locations. Each protection strategy was analysed as a function of the percentage reduction of the first peak of the fault current. Then, taking into account fault events occurring at different places (Faults 1 to 3 in Fig. 1) for each one of these strategies (each of the 31 resulting combinations between the 5 SFCLs depicted in Fig. 1), we have shown that for many of these cases, the recovery time of the superconducting properties for one or more of the installed SFCLs can last over five minutes. This situation jeopardises the making of decisions in favour of investment in this technology (for large scale deployments), due to the associated cooling costs of the SFCLs. However, the integration of a bypass switch parallel to the terminals of the SFCL can successfully reduce the recovery time to a few seconds after the clearance of the fault. Thus, by assuming that the practical operation of the SFCL is assisted by the automatic operation of the BS once the SC material is fully quenched, we have determined that the optimal strategy for the overall protection of a power grid with the topology displayed in Fig. 1, requires a maximum of three SFCLs. The first must be installed at the outgoing feeder of the conventional power plant (SFCL 1), the second must be installed at the outgoing feeder of the wind farm (SFCL 2), and the last one must be connected to the bus-tie coupling the domestic and industrial networks (SFCL 5).

\section{Acknowledgement}

This work was supported by the Engineering and Physical Sciences Research Council (EPSRC) project NMZF/064. X. Zhang acknowledges a grant from the China Scholarship Council (No. 201408060080).

[1] M. Panteli and D. S. Kirschen, "Situation awareness in power systems: Theory, challenges and applications," Electr. Pow. Syst. Res., vol. 122, pp. 140-151, 2015. DOI: 10.1016/j.epsr.2015.01.008

[2] K. M. Muttaqi, J. Aghaei, V. Ganapathy, and A. E. Nezhad, "Technical challenges for electric power industries with implementation of distribution system automation in smart grids," Renew. Sust. Energ. Rev., vol. 46, p. 129-142, 2015. DOI: 10.1016/j.rser.2015.02.013

[3] A. Moreno-Muñoz, J.J. González de la Rosa, J.M. FloresArias, F.J. Bellido-Outerino, and A. Gil-de-Castro, "Energy efficiency criteria in uninterruptible power supply selection," Appl. Energ. Rev., vol. 88, p. 1312-1321, 2011. DOI: 10.1016/j.apenergy.2010.08.017

[4] M. J. Kim, K. Park, K.-Y. Ahn, Y.-G. Kim, and D.-K. Lim, "Application of Fault Current Limiter in 22.9 kV KEPCO grid," 3rd International Conference on Electric Power Equipment Switching Technology (ICEPE-ST), 2015 Busan, Korea., pp. 557-560. DOI: 10.1109/ICEPE-ST.2015.7368376

[5] M. Young, A. Dimitrosvski, Z. Li, Y. Liu, and R. patterson, "Continously Variable Series Reactor: Impacts on Distance Protection using CCVTs," 2015 IEEE Power $\mathcal{E}$ Energy Society General Meeting, pp. 1-5, 2015. DOI: 10.1109/PESGM.2015.7286460

[6] J.-C. Llambes, D. W. Hazelton, C. S. Weber, "Recovery under load performance of 2 nd generation HTS superconducting fault current limiter for electric power transmission lines," IEEE Trans. Appl. Supercond., vol. 19, pp. 1968-1971, 2009.

[7] L. Kovalsky, X. Yuan, K. Tekletsadik, A. Keri, J. Bock, and F. Breuer, "Applications of superconducting fault current limiters in electric power transmission systems," IEEE Trans. Appl. Supercond., vol. 15, pp. 2130-2133, 2005.

[8] L. Ye, M. Majoros, T. Coombs, and A. Campbell, “ System studies of the superconducting fault current limiter in electrical distribution grids," IEEE Trans. Appl. Supcond., vol. 17, pp. 2339-2342, 2007.

[9] B. C. Sung, D. K. Park, J.-W. Park, and T. K. Ko, "Study on a series resistive SFCL to improve power system transient stability: modeling, simulation, and experimental verification," IEEE Trans. Ind. Electron., vol. 56, pp. 2412-2419, 2009.

[10] W. -J. Park, B. C. Sung, and J. -W. Park, "The effect of SFCL on electric power grid with wind-turbine generation system," IEEE Trans. Appl. Supercond., vol. 20, pp. 1177-1181, 2010.

[11] M. Fotuhi-Firuzabad, F. Aminifar, and I. Rahmati, " Reliability study of HV substations equipped with the fault current limiter," IEEE Trans. Power Del., vol. 27, pp. 610-617, 2012.

[12] H. S. Ruiz, X. Zhang, and T. A. Coombs, "Resistive-Type Superconducting Fault Current Limiters: Concepts, Materials, and Numerical Modeling," IEEE Trans. Appl. Supercond., vol. 25, p. 5601405-5601410, 2015. DOI: 10.1109/TASC.2014.2387115

[13] "EEGI R\&I Roadmap 2013-2022", European Electricity Grid Initiative, January, 2013. "UK Electricity Networks," Parliamentary Office of Science and Technology, number 163, October, 2001.

[14] For more information about Crystal Rig II, visit the Fred. Olson Renewables webpage, http://www. fredolsen-renewables.no/overview 
[15] M. Noe and M. Steurer, "High-temperature superconductor fault current limiters: concepts, applications, and development status," Supercond. Sci. Technol., vol. 20, p. R15-R29, 2007.

[16] W. -J Park, B. C. Sung, K. -B. Song, J. -W. Park, "Parameter optimization of SFCL with wind-turbine generation system based on its protective coordination," IEEE Trans. Appl. Supercond., vol. 21, pp. 2153-2156, 2011.

[17] S.-M. Cho, H.-S. Shin, and J.-C. Kim, "Study on coordination of protective relays between primary feeder and interconnecting transformer grounded by SFCL of wind Farm," IEEE Trans. Appl. Supercond., vol. 22, pp. 5500404-5500407, 2012.

[18] B. C. Sung, D. K. Park, J.-W. Park, and T. K. Ko, " Study on optimal location of a resistive SFCL applied to an electric power grid," IEEE Trans. Appl. Supercond., vol. 19, pp. 2048-2052, 2009.

[19] S. Alaraifi, M. E. Moursi, and H. Zeineldin, "Optimal allocation of HTS-FCL for power system security and stability enhancement," IEEE Trans. Power Syst., vol. 28, pp. 4701-4711, 2013.

[20] C. Lacroix and F. Sirois, "Concept of a current flow diverter for accelerating the normal zone propagation velocity in $2 \mathrm{G}$ HTS coated conductors," Supercond. Sci. Technol., vol. 27, p. $035003,2014$.

[21] S. M. Blair, C. D. Booth, and G. M. Burt, "Current-time characteristics of resistive superconducting fault current limiters," IEEE Trans. Appl. Supercond. vol. 22, p. 5600205, 2012.

[22] R. Wesche, "Temperature dependence of critical currents in superconducting Bi-2212/Ag wires," Physica C, vol. 246, pp. 186194, 1995.

[23] M. F. Crommie and A. Zettl, "Thermal-conductivity anisotropy of single-crystal $\mathrm{Bi}_{2} \mathrm{Sr}_{2} \mathrm{CaCu}_{2} \mathrm{O}_{8}$," Phys. Rev. B, vol. 43, 408412, 1991.

[24] J. Bock, F. Breuer, H. Walter, M. Noe, R. Kreutz, M. Kleimaier, K. H. Weck, and S. Elschener, "Development and successful testing of MCP BSCCO-2212 components for a 10 MVA resistive superconducting fault current limiter," Supercond. Sci. Technol., vol. 17, p. S122, 2004.

[25] E. J. Cukauskas and L. H. Allen, "Critical current characteristics of composite thin films of Au and YBa2Cu3O7," Physica C, vol. 313, pp. 11-20, 1999.

[26] S. R. Currás, J. Viña, M. Ruibal, M. T. González, M. R. Osorio, J. Maza, J. A. Veira, and F. Vidal, "Normal-state resistivity versus critical current in $\mathrm{YBa} 2 \mathrm{Cu} 3 \mathrm{O} 7-\mathrm{d}$ thin films at high current densities," Physica C, vol. 372-376, pp. 1095-1098, 2002.

[27] Z. Melhem, "High temperature superconductors (HTS) for energy applications," Woodhead Publishing Ltd, 2011.

[28] C. Goodhand, "Northern Powergrid: 33kV Superconducting Fault Current Limiter, LCN Fund Project Final Closedown Report," Northern Powergrid, January 2015.

[29] R. M. Strzelecki, "Power electronics in smart electrical energy networks," Springer Science $\mathcal{E}$ Business Media, 2008.

[30] C. Neumann, "Superconducting Fault Current Limiter (SFCL) in the Medium and High Voltage Grid," IEEE Power Engineering Society General Meeting, 2006. 